\title{
The Left-Right Symmetry Breaking Mechanism for the New Left-Right Symmetry Model
}

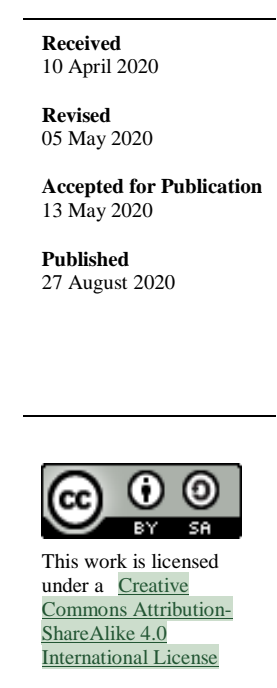

A Ferdiyan ${ }^{1 *}$, A S Adam ${ }^{2}$, and M Satriawan ${ }^{3}$

1. Department of Physics, Faculty of Mathematics and Natural Sciences, Universitas Jenderal Soedirman, Karangwangkal, Purwokerto, 53123, Indonesia.

2. Study Program of Physics, Faculty of Mathematics and Natural Sciences, Universitas Pertahanan Indonesia, Sentul IPSC Area, Bogor, 16810, Indonesia.

3. Department of Physics, Faculty of Mathematics and Natural Sciences, Universitas Gadjah Mada, Bulaksumur, Yogyakarta, 55281, Indonesia.

*E-mail: aferdiyan@unsoed.ac.id

\begin{abstract}
We added a mechanism for breaking the left-right (LR) symmetry in the new variant of the left-right symmetry model. We added to the modela new scalar field $\phi$ that transforms oddly under the new LR transformation. The mechanism can provide different values for the vacuum expectation values of the left and right Higgs scalar, $v_{L}$, and $v_{R}$. A simple numerical analysis was performed to make sure that the parameters space in this model can satisfy some constraints of the model. It is shown that for some parameter values, this mechanism can fulfill the constraint of $v_{L}=246 \mathrm{GeV}$ and the ratio $v_{R} / v_{L}>30$.

Keywords: Left-right symmetry, left-right symmetry breaking, standard model extension
\end{abstract}

\section{Introduction}

The left-right symmetry model (LRSM) as an extension of the standard model (SM) of particle physics has received much attention from scholars in recent years. The reason is due to the possibility of accessing the phenomenology of the LRSM at the LHC (Large Hadron Collider) after the energy upgrade [1], [2]. The LRSM is attractive because of several reasons, such as the seesaw mechanism for neutrino mass and P-symmetry between the left and right Gauge sectors at high energies [3]-[5].

One of the variants of the LRSM is a model introduced by [6], which will be called a dual LR model in this article. This model has an additional global quantum number $F$ and leptoquarks in it, which is different from the usual LRSM. The quantum number $F$ with the left-right (LR) symmetry makes it possible for the neutrino to get mass from a single seesaw mechanism, but it also forces all neutrinos to be Dirac neutrinos. There is no Majorana neutrino in this model, which means that there is also no leptogenesis. The baryon asymmetry can be explained by using the decay of leptoquarks into SM particles.

The experimental fact observed until now shows that the right sector must be decoupled from the left sector since there is no detection of right charged Gauge-Bosons $W_{R}^{ \pm}$until today [7], [8]. In the SM, there is only left charged Gauge-Bosons $W_{L}^{ \pm}$present, as the SM are a left-right asymmetric model which only has V-A current [9], [10]. The absence of $W_{R}^{ \pm}$means that at the current energy level LR symmetry must be broken. There are several ways to facilitate such a breaking, and in [6], we only mention a soft breaking term that breaks the LR symmetry. It is necessary to explore further about the LR symmetry breaking in the dual LR model since it can give more understanding about the phenomenological aspect of the model. In this paper, we propose one possible scenario that can lead to the soft breaking term for the dual LR model. A more detail explanation of the LR symmetry breaking mechanism of the dual LR model is done in the following sections. 
Table 1. Particle content of the model, with their quantum number assignment.

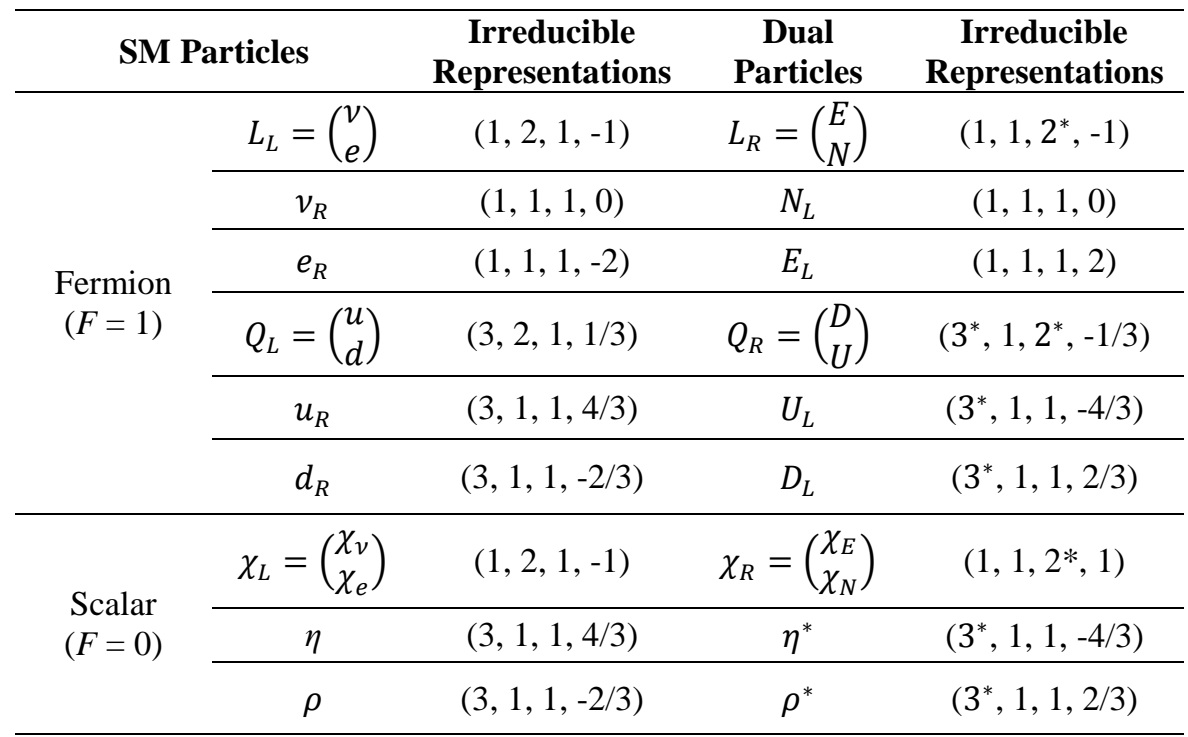

\section{Method}

\subsection{Description of the Dual LR Model}

The model is based on the Gauge group $S U(3) \otimes S U(2)_{L} \otimes S U(2)_{R} \otimes U(1)$, but with a new LR transformation, which reverses both the LR chirality and the local quantum number of a particle. The LR chirality and Gauge group of a particle were changed into its charge conjugate representation. In addition, the $S U(2)_{L}$ representation was changed into $S U(2)_{R}$ representation, and vice versa. The fermion and the scalar contents of the model are displayed in Table 1, categorized as SM particles and their dual LR particles. These dual LR particles were related to the SM particles by the new LR transformation.

The Gauge couplings of $S U(2)_{L}$ and $S U(2)_{R}$ in this model are equal, $g_{L}=g_{R}=g$. The scalar sector consists of the two Higgs doublet $\chi_{L}$ and $\chi_{R}$, and also two leptoquarks scalar $\eta$ and $\rho$ with their corresponding dual LR particles. The leptoquarks were introduced to facilitate the decay of massive dual LR particles into the SM particles and neutrinos. The existence of leptoquarks is necessary for this model not only for the decay process of the dual LR particles but also to generate baryon number asymmetry.

There are several advantages of this model, such as that there is no mixing between the left and right Gauge-Bosons since there is no bidoublet in this model. Dual LR model can also provide a formulation for neutrino oscillation, has no proton decay, and can avoid the big bang nucleosynthesis (BBN) constraint. Baryogenesis from the decay of leptoquarks particles can lead to baryon number asymmetry in the universe (BAU). All of the dual LR particles have large mass and should have decayed at the current low energy level. [6].

\subsection{LR Symmetry in the Scalar Sectors}

The scalar sector of the LRSM has been discussed in several papers in detail, particularly the breaking of charge (C) and/or parity (P) in such models [1], [11]-[15]. In the dual LR model, the scalar potential that is invariant under the Gauge group and the new LR transformation is given by

$$
\begin{aligned}
V= & -\mu_{L}^{2}\left|\chi_{L}\right|^{2}-\mu_{R}^{2}\left|\chi_{R}\right|^{2}+\mu_{1}^{2}|\eta|^{2}+\mu_{2}^{2}|\rho|^{2}+\lambda_{1}\left(\left|\chi_{L}\right|^{4}+\left|\chi_{R}\right|^{4}\right)+\lambda_{2}|\eta|^{4}+\lambda_{3}|\rho|^{4} \\
& +\epsilon_{1}|\eta|^{2}\left(\left|\chi_{L}\right|^{2}+\left|\chi_{R}\right|^{2}\right)+\epsilon_{2}|\rho|^{2}\left(\left|\chi_{L}\right|^{2}+\left|\chi_{R}\right|^{2}\right)+\epsilon_{3}|\eta|^{2}|\rho|^{2}+\epsilon_{4}\left|\chi_{L}\right|^{2}\left|\chi_{R}\right|^{2} \\
& +\epsilon_{5} \rho^{\dagger} \eta \eta^{\dagger} \rho
\end{aligned}
$$

with the parameters $\mu_{i}, \lambda_{i}$ and $\epsilon_{i}$. These are scalar constants, which can be chosen so that $\chi_{L}$ and $\chi_{R}$ have non zero vacuum expectation values (VEV). The leptoquarks $\eta$ and $\rho$ must have zero VEV since otherwise, the gluon tends be massive. 
The doublet Higgs $\chi_{L}$ and $\chi_{R}$ can have different VEV, and can be written as

$$
\left\langle\chi_{L}\right\rangle=\left(\begin{array}{c}
v_{L} \\
0
\end{array}\right),\left\langle\chi_{R}\right\rangle=\left(\begin{array}{c}
0 \\
v_{R}
\end{array}\right)
$$

where in general $v_{L} \neq v_{R}$. Dual LR model then only has two Higgs doublet with non-vanishing VEV. By inserting the VEV of all scalar particles into the potential and minimizing it, the resulting two sets of the equation are given by

$$
\begin{aligned}
& -2 \mu_{L}^{2} v_{L}+4 \lambda_{1} v_{L}^{3}+2 \epsilon_{4} v_{L} v_{R}^{2}=0 \\
& -2 \mu_{R}^{2} v_{R}+4 \lambda_{1} v_{R}^{3}+2 \epsilon_{4} v_{R} v_{L}^{2}=0
\end{aligned}
$$

The value of $v_{L}$ and $v_{R}$ in terms of $\mu_{i}, \lambda_{i}$ and $\epsilon_{i}$ are given by solving equation (3) and (4) together

$$
v_{L}=\sqrt{\frac{\mu_{R}^{2} \epsilon_{4}-2 \mu_{L}^{2} \lambda_{1}}{\epsilon_{4}^{2}-4 \lambda_{1}^{2}}}, v_{R}=\sqrt{\frac{\mu_{L}^{2} \epsilon_{4}-2 \mu_{R}^{2} \lambda_{1}}{\epsilon_{4}^{2}-4 \lambda_{1}^{2}}}
$$

It can already be seen that in this model the VEV of left and right Higgs doublet, $v_{L}$, and $v_{R}$ are symmetric with respect to the LR symmetry. As a consequence, their value should not be too different if $\mu_{L}$ and $\mu_{R}$ have similar values. This can become a problem, since the mass of $W_{R}^{ \pm}$is proportional to $v_{R}$, i.e., $M_{W_{R}}=\frac{1}{4} g v_{R}^{2}$. If $v_{R} \approx v_{L}$, then $W_{R}^{ \pm}$should have been found in the LHC experiments.

\section{Results and Discussion}

\subsection{LR Symmetry Breaking}

The LR symmetry must be broken in order to give different values to $v_{L}$ and $v_{R}$. The value of $v_{R}$ must be relatively high to explain the no detection of $W_{R}^{ \pm}$. One way to break the LR symmetry is by introducing an additional scalar singlet field (see, for example [16]-[18]), $\phi$ with the quantum number $(1,1,1,0)$, which under the LR transformation transforms as $\phi \rightarrow-\phi$. With the addition of $\phi$, the scalar potential given in (1) changes into

$$
\begin{aligned}
V= & -\mu_{1}^{2}|\phi|^{2}-\mu_{2}^{2}\left(\left|\chi_{L}\right|^{2}+\left|\chi_{R}\right|^{2}\right)+\mu_{3}^{2}|\eta|^{2}+\mu_{4}^{2}|\rho|^{2}+\lambda_{1}|\phi|^{4}+\lambda_{2}\left(\left|\chi_{L}\right|^{4}+\left|\chi_{R}\right|^{4}\right) \\
& +\lambda_{3}|\eta|^{4}+\lambda_{4}|\rho|^{4}+\epsilon_{1}|\phi|^{2}\left(\left|\chi_{L}\right|^{2}+\left|\chi_{R}\right|^{2}\right)+\epsilon_{2}|\eta|^{2}\left(\left|\chi_{L}\right|^{2}+\left|\chi_{R}\right|^{2}\right) \\
& +\epsilon_{3}|\rho|^{2}\left(\left|\chi_{L}\right|^{2}+\left|\chi_{R}\right|^{2}\right)+\epsilon_{4}|\phi|^{2}|\eta|^{2}+\epsilon_{5}|\phi|^{2}|\rho|^{2}+\epsilon_{6}|\eta|^{2}|\rho|^{2}+\epsilon_{7}\left|\chi_{L}\right|^{2}\left|\chi_{R}\right|^{2} \\
& +\epsilon_{8} \rho^{\dagger} \eta \eta^{\dagger} \rho+\left(\delta_{1} \phi\left(\left|\chi_{L}\right|^{2}-\left|\chi_{R}\right|^{2}\right)+\text { h. c. }\right)
\end{aligned}
$$

Notice that the parameters for $\chi_{L}$ and $\chi_{R}$ in the above potential are the same, i.e., the mass term $\mu_{2}$. It is assumed that at this scale, the LR symmetry is still present. There is a new term which will be responsible for the LR symmetry breaking, i.e., the term with the $\delta_{1}$ factor. This term explicitly breaks the symmetry, which can be seen from the minus sign in it, as a consequence of the transformation of $\phi$ that transforms oddly under the LR transformation. The singlet $\phi$ can have a VEV, and it can be written as

$$
\langle\phi\rangle=v_{h}
$$

Inserting this value together with the VEV for $\chi_{L}, \chi_{R}, \rho$, and $\eta$ into equation (6), and minimizing it with respect to $v_{L}$ and $v_{R}$ gives the following result

$$
\begin{aligned}
& -2 \mu_{2}^{2} v_{L}+4 \lambda_{2} v_{L}^{3}+2 \epsilon_{1} v_{h}^{2} v_{L}+2 \epsilon_{7} v_{L} v_{R}^{2}+2 \delta_{1} v_{h} v_{L}=0 \\
& -2 \mu_{2}^{2} v_{R}+4 \lambda_{2} v_{R}^{3}+2 \epsilon_{1} v_{h}^{2} v_{R}+2 \epsilon_{7} v_{R} v_{L}^{2}-2 \delta_{1} v_{h} v_{R}=0
\end{aligned}
$$

Now the value of $v_{L}$ and $v_{R}$ can be obtained by solving equation (8) and (9) together:

$$
\begin{aligned}
& v_{L}=\sqrt{\frac{\left(\mu_{2}^{2}-\epsilon_{1} v_{h}^{2}\right)}{2 \lambda_{2}+\epsilon_{7}}-\frac{\delta_{1} v_{h}}{2 \lambda_{2}-\epsilon_{7}}} \\
& v_{R}=\sqrt{\frac{\left(\mu_{2}^{2}-\epsilon_{1} v_{h}^{2}\right)}{2 \lambda_{2}+\epsilon_{7}}+\frac{\delta_{1} v_{h}}{2 \lambda_{2}-\epsilon_{7}}}
\end{aligned}
$$




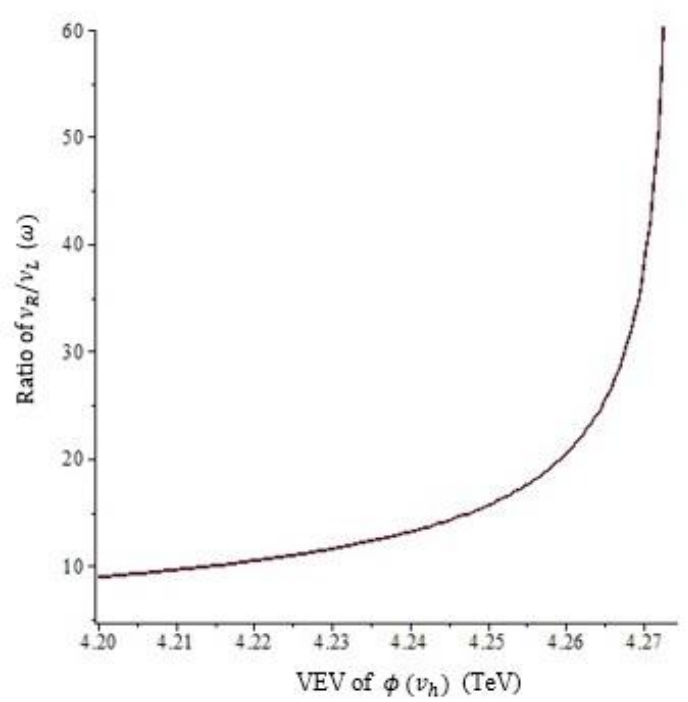

(a)

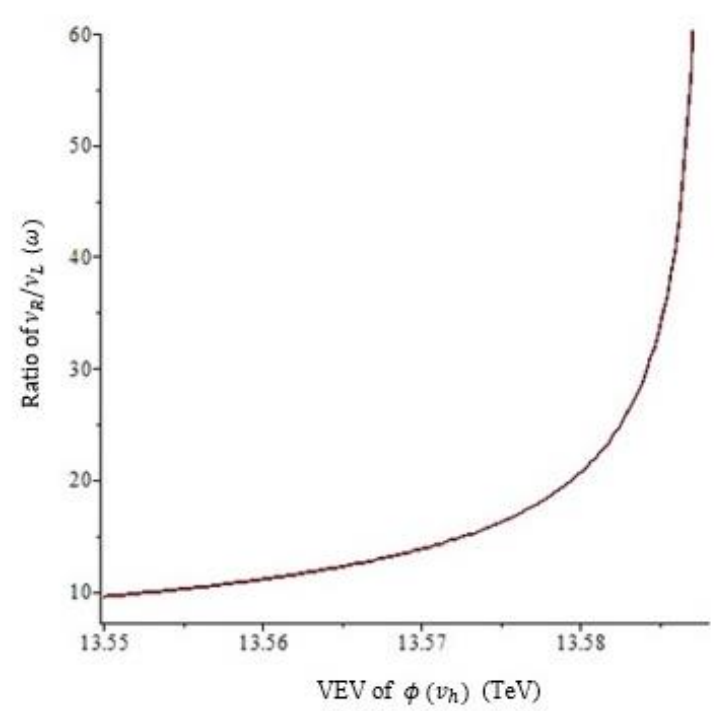

(b)

Figure 1. (a) Ratio of $v_{R} / v_{L}(\omega)$ as function of VEV of $\phi\left(v_{h}\right)$ (with $\mu_{2}^{2}=100 \mathrm{TeV}$ and $\delta_{1}=6.374025316 \mathrm{TeV}$ ), (b) Ratio of $v_{R} / v_{L}(\omega)$ as function of VEV of $\phi\left(v_{h}\right)\left(\right.$ with $\mu_{2}^{2}=250 \mathrm{TeV}$ and $\left.\delta_{1}=2.402889173 \mathrm{TeV}\right)$.

Depending on the value of $\mu_{2}, \lambda_{2}, \epsilon_{1}$, and $\epsilon_{7}$, the term with $\delta_{1}$ can make the difference between $v_{L}$ and $v_{R}$ to be sufficiently large. To explore further, their values can be taken based on some assumptions that (i) $\mu_{2}^{2}$ is related to the LR symmetry, and it may as well be assumed to be above the LHC energy. Current energy reach of LHC is $14 \mathrm{TeV}$, then it will be assumed that $\mu_{2}$ is of the order of $\left(10^{2}\right) \mathrm{TeV}$; (ii) $\epsilon_{7}, \epsilon_{1}$, and $\lambda_{2}$ are constant parameters related to the interaction between scalar fields, with the order of unity; and (iii) $\delta_{1}$ is a constant parameter related to $\phi$ and it will be determined from each test case values of $\mu_{2}^{2}$.

There are two bounds that will be checked in order to determine the values of the VEVs. The first is that $v_{L}$ must give the experimental value of $246 \mathrm{GeV}$, and the second comes from the minimum bound for $\omega=v_{R} / v_{L}$, the ratio between left and right VEV [6], [19], [20]. An analysis has been done for two choices of values, and the result is given in Figure 1.a and 1.b.

The $\delta_{1}$ values are obtained by inserting $\mu_{2}^{2}$ into equation (10) and (11) along with other parameters as described in the assumptions. Then it is solved simultaneously by imposing two conditions, $v_{L}=246 \mathrm{GeV}$ and $\omega=30$. The latter is abound for this model, $\omega>30$, obtained from fitting correction of the SM fermions coupling with a neutral weak boson $Z_{L}$ to the experimental data [19]. The result shows that it is possible to have realistic values for both $\delta_{1}$ and $v_{h}$ within each test case parameters values. The first case with $\mu_{2}^{2}=100 \mathrm{TeV}$ and $\delta_{1}=6.374025316 \mathrm{TeV}$ gives the values of $v_{h}=4.267624258 \mathrm{TeV}$. This $v_{h}$ value exactly gives $\omega=30$, for higher $v_{h}$, the $\omega$ will also increase.

The second case with $\mu_{2}^{2}=250 \mathrm{TeV}$ and $\delta_{1}=2.402889173 \mathrm{TeV}$ that gives the values of $v_{h}=$ $13.58421315 \mathrm{TeV}$. This $v_{h}$ value exactly gives $\omega=30$, and also for higher $v_{h}$, $\omega$ will also increase. We conclude that there is enough parameter space in this model to break the LR symmetry while also satisfying several bounds given by the experimental data. Further works can be done on the phenomenological aspect of the new field $\phi$.

\section{Conclusion}

We have investigated the possibility of breaking the LR symmetry in the dual LR model. The mechanism needs the introduction of a new field into the model, namely a new scalar singlet $\phi$. The addition of $\phi$ can break the symmetry in $v_{L}$ and $v_{R}$ values, when it gains its VEV. The reason underlies behind the LR symmetry breaking is the odd transformation of the new field, $\phi \rightarrow-\phi$ under the LR transformation. This introduces a term that will break the LR symmetry in the scalar potential. Two test cases values have been analyzed numerically, and the result shows that it is possible to have a set of values that gives good agreement with the bounds from the experimental data, i.e. $v_{L}=246 \mathrm{GeV}$ and $\omega \geq 30$. 


\section{References}

[1] A. Maiezza, G. Senjanovic, and J. C. Vasquez, "Higgs Sector of the Minimal Left-Right Symmetric Theory," Phys. Rev. D., vol. 95, no. 9, p. 095004, 2017.

[2] G. Senjanovic, "Is Left-Right Symmetry the Key?," Mod. Phys. Lett. A, vol. 32, no. 4, pp. 1-13, 2017.

[3] R. N. Mohapatra and G. Senjanović, "Neutrino Mass and Spontaneous Parity Nonconservation," Phys. Rev. Lett., vol. 44, no. 14, pp. 912-915, 1980.

[4] R. N. Mohapatra and G. Senjanović, "Neutrino Masses and Mixings in Gauge Models with Spontaneous Parity Violation," Phys. Rev. D, vol. 23, no. 1, pp. 165-180, 1981.

[5] G. Senjanović, "Spontaneous Breakdown of Parity in A Class of Gauge Theories," Nucl. Phys. Sect. B, vol. 153, pp. 334-364, 1979.

[6] A. S. Adam, A. Ferdiyan, and M. Satriawan, "A New Left-Right Symmetry Model," Adv. High Energy Phys., vol. 2020, no. 2, pp. 1-9, 2020.

[7] M. Aaboud et al., "Search for Heavy Majorana or Dirac Neutrinos and Right-Handed W Gauge Bosons in Final States with Two Charged Leptons and Two Jets at $\sqrt{ } \mathrm{s}=13 \mathrm{TeV}$ with the ATLAS Detector," J. High Energy Phys., vol. 16, pp. 1-44, 2019.

[8] M. Aaboud et al., "Search for A Right-Handed Gauge Boson Decaying into A High-Momentum Heavy Neutrino and A Charged Lepton in PP Collisions with the ATLAS Detector at $\mathrm{s}=13$ TeV," Phys. Lett. Sect. B Nucl. Elem. Part. High-Energy Phys., vol. 798, p. 134942, 2019.

[9] F. Halzen and A. D. Martin, Quarks and Leptons: An Introductory Course in Modern Particle Physics, New Jersey: John Wiley \& Sons, 1984.

[10] M. E. Peskin and D. V. Schroeder, An Introduction to Quantum Field Theory, Boston: AddisonWesley, 1995.

[11] D. Cocolicchio and G. L. Fogli, "Symmetry-Breaking Patterns in Left-Right-Symmetric Models: How to Ensure Natural Flavor Conservation and A Soft CP Violation," Phys. Rev. D, vol. 32, no. 11, pp. 3020-3036, 1985.

[12] F. Siringo, "Symmetry Breaking of the Symmetric Left-Right Model without A Scalar Bidoublet," Eur. Phys. J. C, vol. 32, no. 4, pp. 555-559, 2004.

[13] P. S. B. Dev et al., "Vacuum Structure of the Left-Right Symmetric Model," J. High Energy Phys., vol. 2019, no. 2, p. 159, 2019.

[14] G. Barenboim et al., "Higgs Sector of the Minimal Left-Right Symmetric Model," Phys. Rev. D - Part. Fields. Gravit. Cosmol., vol. 65, no. 9, p. 095003, 2002.

[15] S. Chakdar et al., "Collider Signatures of Mirror Fermions in the Framework of A Left-Right Mirror Model," Phys. Rev. D - Part. Fields Gravit. Cosmol., vol. 88, no. 9, pp. 1-16, 2013.

[16] M. Satriawan, "CP-Mirror Extension of Standard Model in $\mathrm{SU}(3) \otimes \mathrm{SU}(2)_{\mathrm{L}} \otimes \mathrm{SU}(2)_{\mathrm{R}} \otimes$ $\mathrm{U}(1)_{\mathrm{I}}$," in DESY Theory Workshop - Cosmology Meets Particle Physics, Hamburg: Ludwig Maximilians Universitat, 2011.

[17] A. Ferdiyan, "Neutrino Singlet dalam Model Cermin CP sebagai Kandidat Materi Gelap," M.Sc. thesis, Department of Physics, Faculty of Mathematics and Natural Sciences, Universitas Gadjah Mada, Yogyakarta, 2012.

[18] A. S. Adam, "Bariogenesis dalam Model Cermin CP," M.Sc. thesis, Department of Physics, Faculty of Mathematics and Natural Sciences, Universitas Gadjah Mada, Yogyakarta, 2012.

[19] Y. A. Coutinho, J. A. M. Simões, and C. M. Porto, "Fermion Masses in A Model for Spontaneous Parity Breaking," Eur. Phys. J. C, vol. 18, no. 4, pp. 779-784, 2001.

[20] F. M. L. D. Almeida et al., "Double Seesaw Mechanism in A Left-Right Symmetric Model with TeV Neutrinos," Phys. Rev. D - Part. Fields, Gravit. Cosmol., vol. 81, no. 5, pp. 1-7, 2010. 